\title{
OPEN High expression of tight junction protein 1 as a predictive biomarker for bladder cancer grade and staging
}

\author{
Yi-Chen Lee $\mathbb{(}^{1,2}$, Kuo-Wang Tsai $\oplus^{3}$, Jia-Bin Liao ${ }^{4}$, Wei-Ting Kuo ${ }^{5,6}$, Yu-Chan Chang ${ }^{7}$ \& \\ Yi-Fang Yang $\mathbb{1}^{8} \bowtie$
}

Tight junction proteins 1-3 (TJP1-3) are components of tight junctions that can link transmembrane proteins to the actin cytoskeleton, and their incidence directly correlates to metastasis. However, the role of the TJP family in bladder cancer has not been adequately evaluated. In this study, we evaluated the genetic changes, mRNA and protein expressions of the target genes of the TJP family in bladder cancer patients using online database and immunohistochemistry, respectively. We found that TJP1 was amplified in bladder cancer tissue and that the protein expression levels were significantly associated with age $(p=0.03)$, grade $(p=0.007)$, and stage $(p=0.011)$. We also examined the correlation between TJP1 and other high-frequency mutation genes using TIMER. TJP1 mRNA levels were positively correlated with TTN and RYR3 mRNA levels in bladder cancer tissue. Taken together, $T J P 1$ expression is associated with poor clinical outcomes in patients with bladder cancer and can be a useful predictive biomarker for bladder cancer staging.

Bladder cancer is the common cause of cancer-related deaths worldwide ${ }^{1,2}$. In 2019, there were 30,543 cancerrelated deaths in males in Taiwan, of which 711 deaths $(2.3 \%)$ were from bladder cancer. Bladder cancers are classified into non-muscle-invasive bladder cancer (NMIBC) and muscle-invasive bladder cancer (MIBC) ${ }^{3}$. Urothelial carcinoma is the most common type of bladder cancer. Approximately $50-70 \%$ of patients with NMIBC show recurrence after treatment and of those, $10-15 \%$ of patients develop MIBC, which are invasive malignant tumors that have a $50-60 \%$ survival rate (5-year) ${ }^{4,5}$. When the MIBC progresses into metastatic bladder cancer, the 5 -year survival rate of patients is significantly reduced ${ }^{4}$. Locally advanced or metastatic bladder cancer has a high mortality rate, having only a 5\% 5-year survival rate in patients with bladder cancer in the last stage $^{6,7}$. Furthermore, tumor grade as an important prognostic indicator in NMIBC and increasing tumor grade was associated with higher disease progression and recurrence rates ${ }^{1}$. However, the operative mechanisms of how cancer cells modulate the malignant phenotype underlying the disease remain unclear and must be further investigated to effectively improve the poor prognosis of bladder cancer.

Tight junctions (TJs), adherens junctions (AJs), and desmosomes are protective barriers for epithelial and endothelial cells that serve as sentries in the living system ${ }^{8-10}$. TJ barrier functions include the regulation of intercellular communication and paracellular transport ${ }^{11}$. TJ proteins include claudins, occludins, and framework forming proteins: cinguline, PALS1 (protein associated with Lin Seven 1), MUPP1 (multi-PZD domain protein 1), and ZO-1 (TJP1), ZO-2 (TJP2), and ZO-3 (TJP3) (zona occludens) ${ }^{12}$. ZO proteins play an important role in the formation of tight junctions that directly interact with the PDZ domain (ZO-1 or ZO-2) and the $\mathrm{C}$-terminus of claudins ${ }^{13}$. Moreover, these proteins modulate several signaling pathways in cancer cells. Previous studies show that the downregulation of ZO-1 leads to increased motility in pancreatic cancer ${ }^{14}$. However,

\footnotetext{
${ }^{1}$ Department of Anatomy, School of Medicine, College of Medicine, Kaohsiung Medical University, Kaohsiung, Taiwan. 'Department of Medical Research, Kaohsiung Medical University Hospital, Kaohsiung, Taiwan. ${ }^{3}$ Department of Research, Taipei Tzu Chi Hospital, Buddhist Tzu Chi Medical Foundation, New Taipei City, Taiwan. ${ }^{4}$ Department of Pathology and Laboratory Medicine, Kaohsiung Veterans General Hospital, Kaohsiung, Taiwan. ${ }^{5}$ Division of Urology, Department of Surgery, Kaohsiung Veterans General Hospital, Kaohsiung, Taiwan. ${ }^{6}$ School of Medicine, National Yang-Ming University, Taipei, Taiwan. ${ }^{7}$ Department of Biomedical Imaging and Radiological Sciences, National Yang Ming Chiao Tung University, Taipei, Taiwan. ${ }^{8}$ Department of Medical Education and Research, Kaohsiung Veterans General Hospital, No. 386, Dajhong 1st Rd., Zuoying Dist., Kaohsiung 81362, Taiwan. ${ }^{\square}$ email: yvonne845040@gmail.com
} 
the upregulation of ZO-1 contributes to the invasion and adhesion of melanoma cells ${ }^{15}$. Accumulating evidence suggests that $\mathrm{ZO}$ proteins play a central role in cancer progression. However, the role of $\mathrm{ZO}$ proteins in bladder cancer has not been elucidated.

In this study, we investigated whether the ZO family (TJP ZO1, ZO-2, and ZO-3, encoded by the TJP1, TJP2, and TJP3 genes, respectively) is associated with the malignant phenotype in bladder cancer. We examined the DNA copy number and mRNA expression of the ZO family in multiple cancer cell lines and cancer patients using online datasets. Based on the analysis of the bioinformation, we identified the target genes and then provided the clinicopathological data for verification relationships in patients with bladder cancer.

\section{Results}

In silico mRNA and DNA profiles of TJP family members in multiple cancer cell lines. By evaluating the mRNA expression of TJP1, TJP2, and TJP3 in 40 different cancer cell lines, we found that TJP1, TJP2, and TJP3 were upregulated in 29, 40, and 12 different cancer cell types, respectively (Fig. 1). Next, by examining the DNA copy numbers of the TJP family in multiple cancer cell lines, we observed that the DNA copy numbers of TJP1, TJP2, and TJP3 were upregulated in 10, 9, and 7 different cancer cell lines, respectively (Fig. 2). However, the DNA copy numbers and mRNA expression levels were not consistent.

In silico genetic alterations of TJP family members in bladder cancer patients. The genetic alterations of the TJP family in bladder cancer, examined using cBioPortal, showed that the genetically altered ratios of TJP1, TJP2, and TJP3 were 3\%,3\%, and 1.7\%, respectively (Fig. 3A). TJP1 and TJP3 had a $2.5-4 \%$ amplification ratio and TJP2 has a $5 \%$ mutation ratio in bladder cancer patients (Fig. 3B-D). While analyzing the frequency of the co-occurrence of genetically altered TJP1-3 in the same specimen, it was found that genetic alterations of the other TJP family members were not significant in bladder cancer patients (Supplementary Table 1). TJP1 genetic amplification was correlated with mRNA expression in patients with bladder cancer (Supplementary Fig. 1A). In addition, we also compared the TJP1 expression between the protein level and the RNA level from the CCLE dataset (https://depmap.org/portal/). Our results found a strong positive correlation in the bladder cancer cell panel (Spearman nonparametric correlation test; correlation coefficient $=0.582 ; p=0.05$, $\mathrm{n}=11$ ) (Supplementary Fig. 1B). Moreover, we further evaluated expression of TJP1 protein in bladder cancer cell lines. The results showed TJP1 protein was upregulated in bladder cancer cells (Supplementary Fig. 1C). A previous study showed that TJP1 expression is correlated with cell motility in bladder cancer cells ${ }^{16}$. Therefore, in this study, we focused on TJP1 for further investigation of bladder cancer.

TJP1 protein up-regulation is associated with poor clinical outcomes in bladder cancer. As shown in Fig. 4A, TJP1 expression in cancer tissues was classified into two groups (low and high) base on cutoff point, which was set at the median. It was found that high TJP1 expression levels in bladder cancer tissues were significantly associated with age $(p=0.03)$, grade $(p=0.007)$, and stage $(p=0.011)$ (Table 1$)$. IHC staining results show that TJP1 was significantly upregulated in bladder cancer specimens compared to normal bladder tissues (Fig. 4B). The results also showed that TJP1 was significantly upregulated in the urothelial carcinoma group in bladder cancer specimens compared to normal bladder tissues (Fig. 4C).

TJP1 expression positive correlates with TTN in bladder cancer patients. It was observed that TTN, RYR3, TRPM1, RB1, ULK4P3, CHRFAM7A, FAN1, and HERC2 were significantly altered in patients with bladder cancer (Fig. 5A). Furthermore, Fig. 5B shows that TJP1 genetically altered co-occurrence in a series of core genes, including TTN, TP53, and RYR3. We further examined the correlation between the mRNA expression of TJP1 and TTN, TP53, and RYR3 by TIMER ${ }^{17}$. The results showed that TJP1 mRNA levels were positively correlated with TTN and RYR3 mRNA levels in bladder cancer tissues (Fig. 5C). Moreover, we examined the protein-protein interactions network by using STRING dataset ${ }^{18}$. The results showed TJP1 interaction with TTN and RYR3 via TP53 (Fig. 5D and Supplementary Table 2).

TJP family expression correlated with chemotherapy response in bladder cancer cells. It has been shown that genetic determinants for chemotherapy and radiotherapy response in bladder cancer ${ }^{19}$. To examine the correlation between TJP family mRNA expression and chemotherapy drugs in bladder cancer cells. We found TJP2 expression was positively correlated with IC50 of cisplatin, and TJP3 expression was positively correlated with IC50 of mitomycin C in bladder cancer cell lines (Table 2 and Supplement Figs. 2-4).

\section{Discussion}

TJP1, also known as zona occludens 1 (ZO-1), is a tight junction protein that can regulate actin cytoskeleton remodeling ${ }^{20}$. Altered expression of TJP1 is found in many cancers and is responsible for modulating cancer migration and invasion ${ }^{15,20-22}$. In this study, the TJP family was evaluated in multiple cancer cell lines and it is a predictive biomarker for bladder cancer staging.

Our data showed that TJP1 is upregulated in multiple cancer cell lines (Fig. 1A). TJP expression regulates several signaling and transcriptional pathways in cancer ${ }^{23}$ and is involved in the epithelial-mesenchymal transition (EMT) associated with tumor invasion ${ }^{24}$. Downregulation of TJP1 expression has been observed in gastrointestinal adenocarcinoma, breast cancer, and colorectal carcinoma ${ }^{25-27}$. TJP1 expression is regulated by E-cadherin in breast carcinoma ${ }^{26}$. During EMT, downregulation of E-cadherin is accompanied by the upregulation of $\mathrm{N}$-cadherin expression, which promotes cell motility and survival advantage in the early stage tumor ${ }^{28,29}$. In contrast, Smalley et al. showed that TJP1 is upregulated and co-localized with N-cadherin and contributes to adhesion and 
A

${ }_{6} \quad \therefore \quad$ TJP1

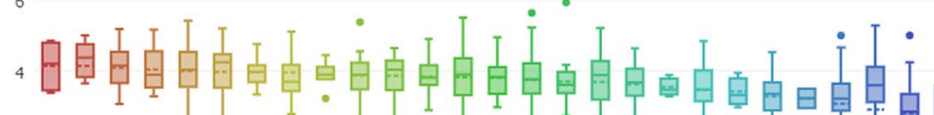

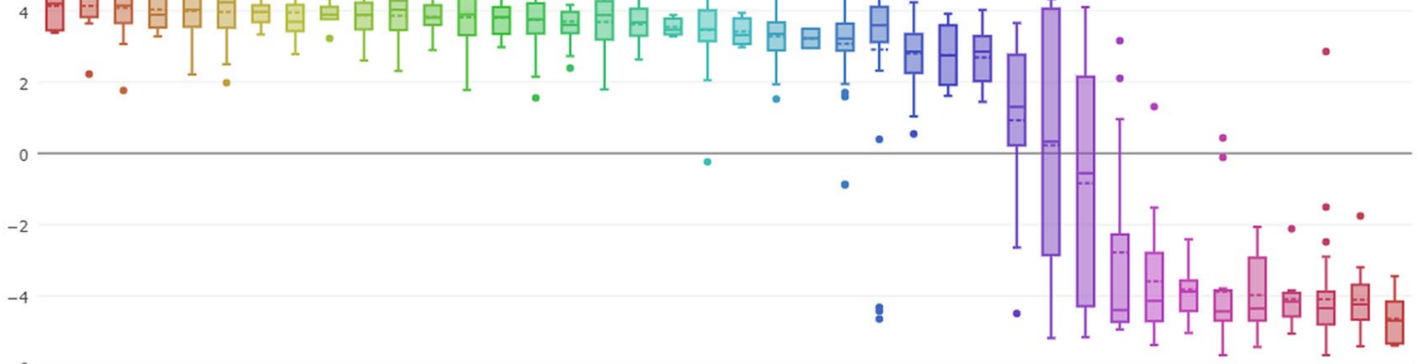

$-6$

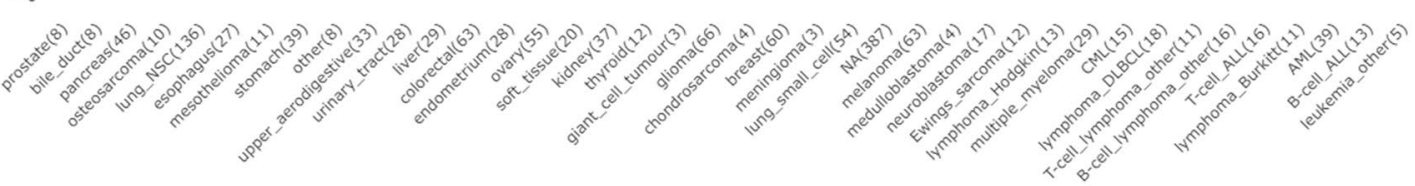

B

TJP2
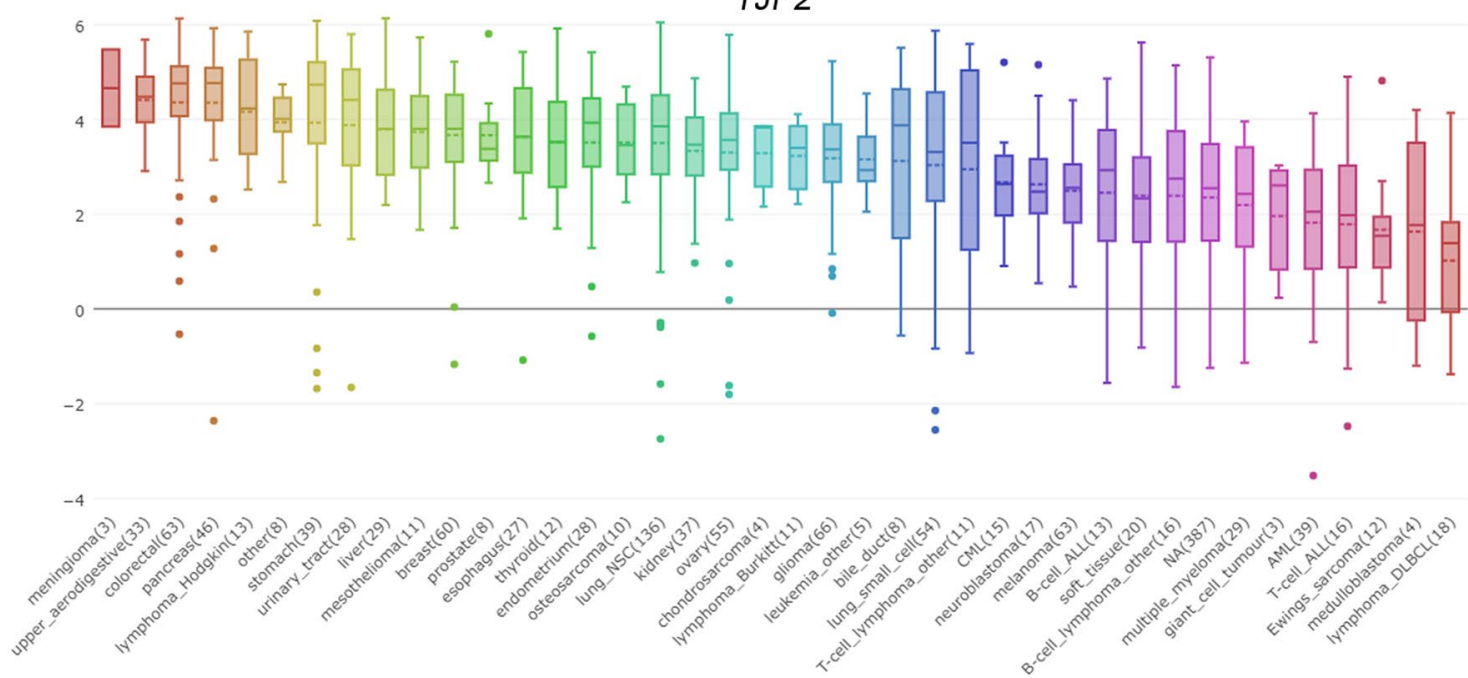

C

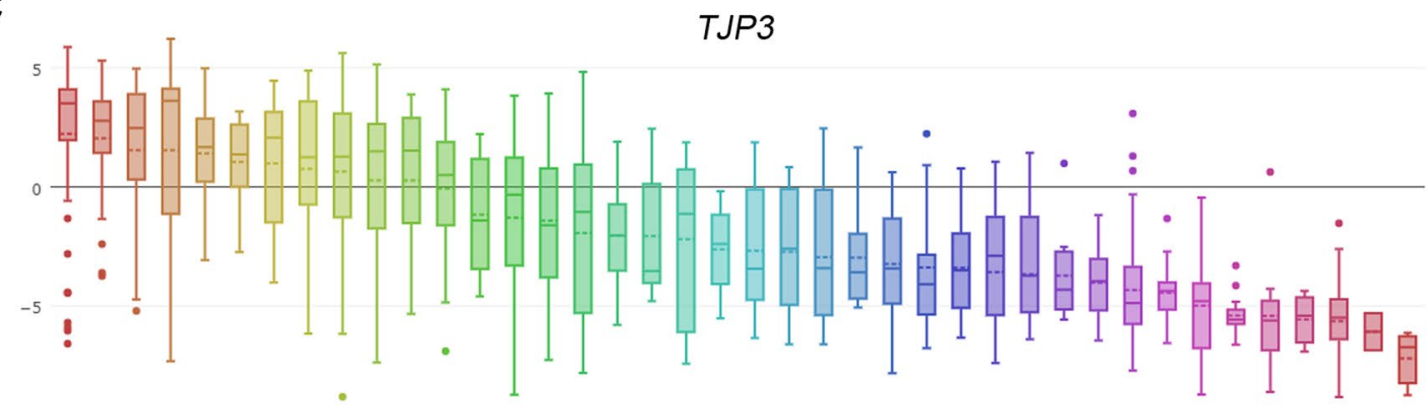

$-10$

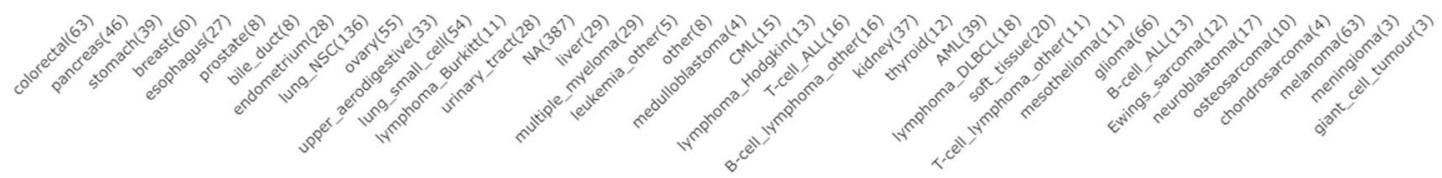

Figure 1. TJP family mRNA expression in multiple cancer cell lines. Relative TJP1 (A), TJP2 (B) and TJP3 (C) mRNA expression in multiple cancer cell lines (CCLE dataset). The number of parentheses that how many cell have in the same cancer type. 
A
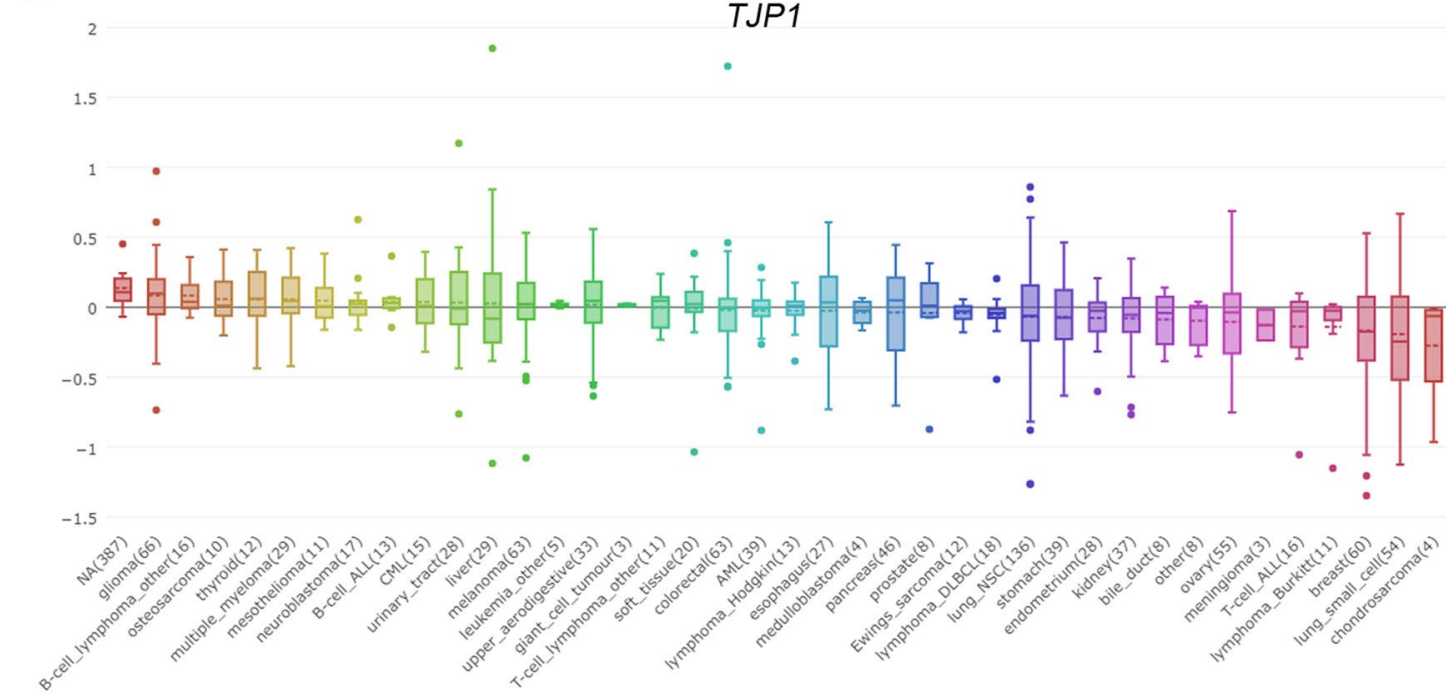

$\mathrm{B}$
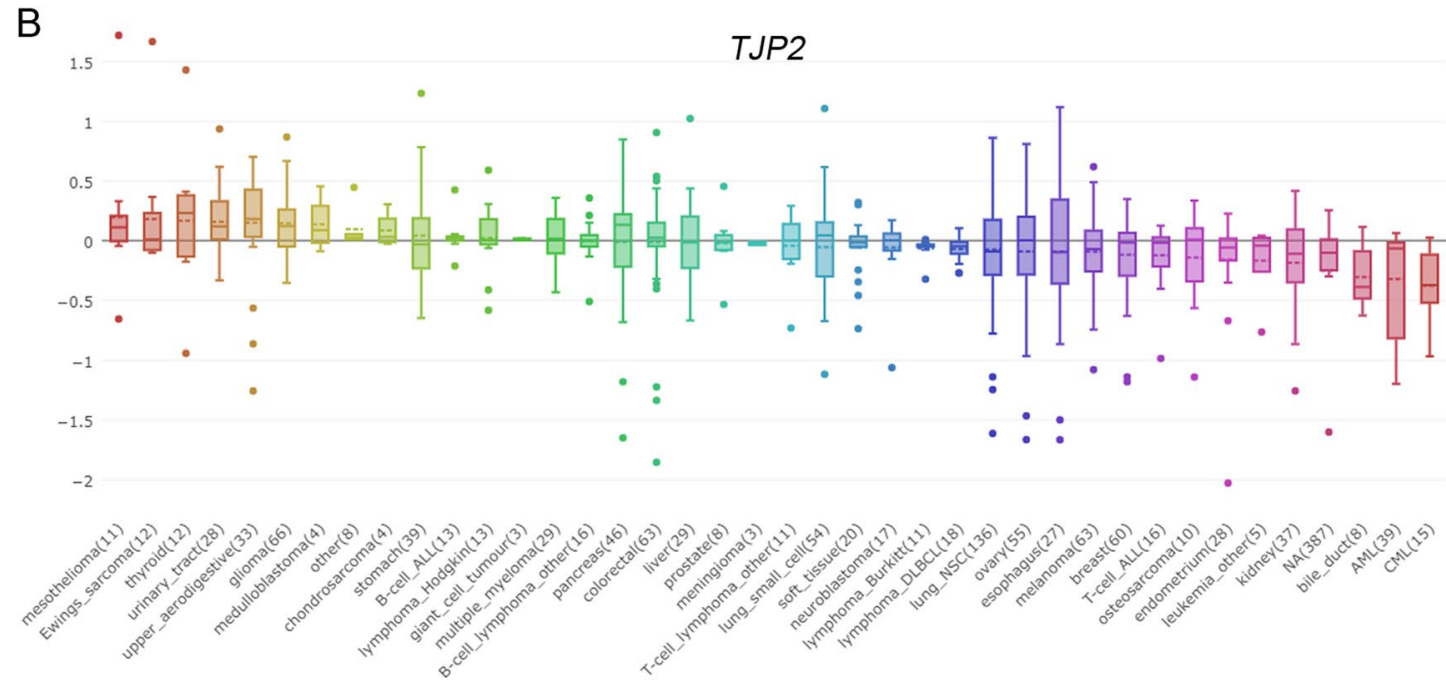

C
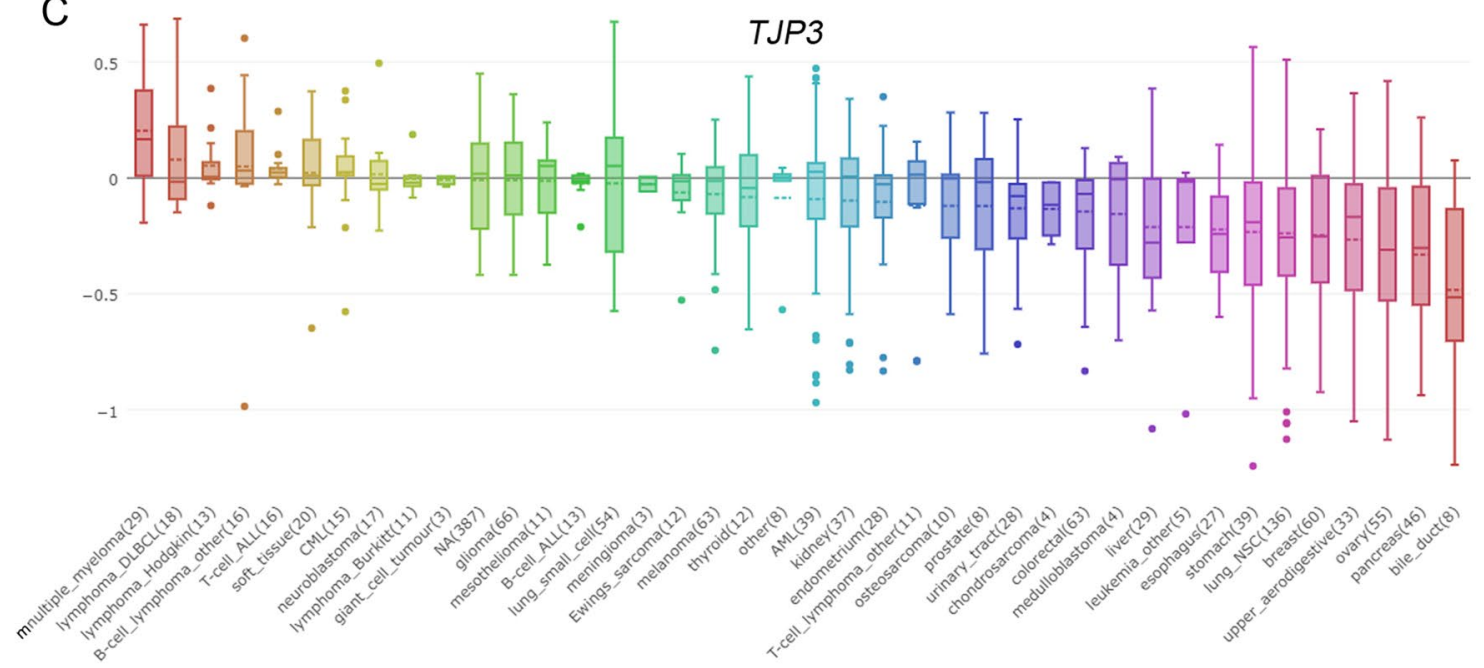

Figure 2. TJP1 DNA copy number in multiple cancer cell lines. Relative TJP1 (A), TJP2 (B) and TJP3 (C) DNA copy number in multiple cancer cell lines (CCLE dataset). The number of parentheses that how many cell have in the same cancer type. 


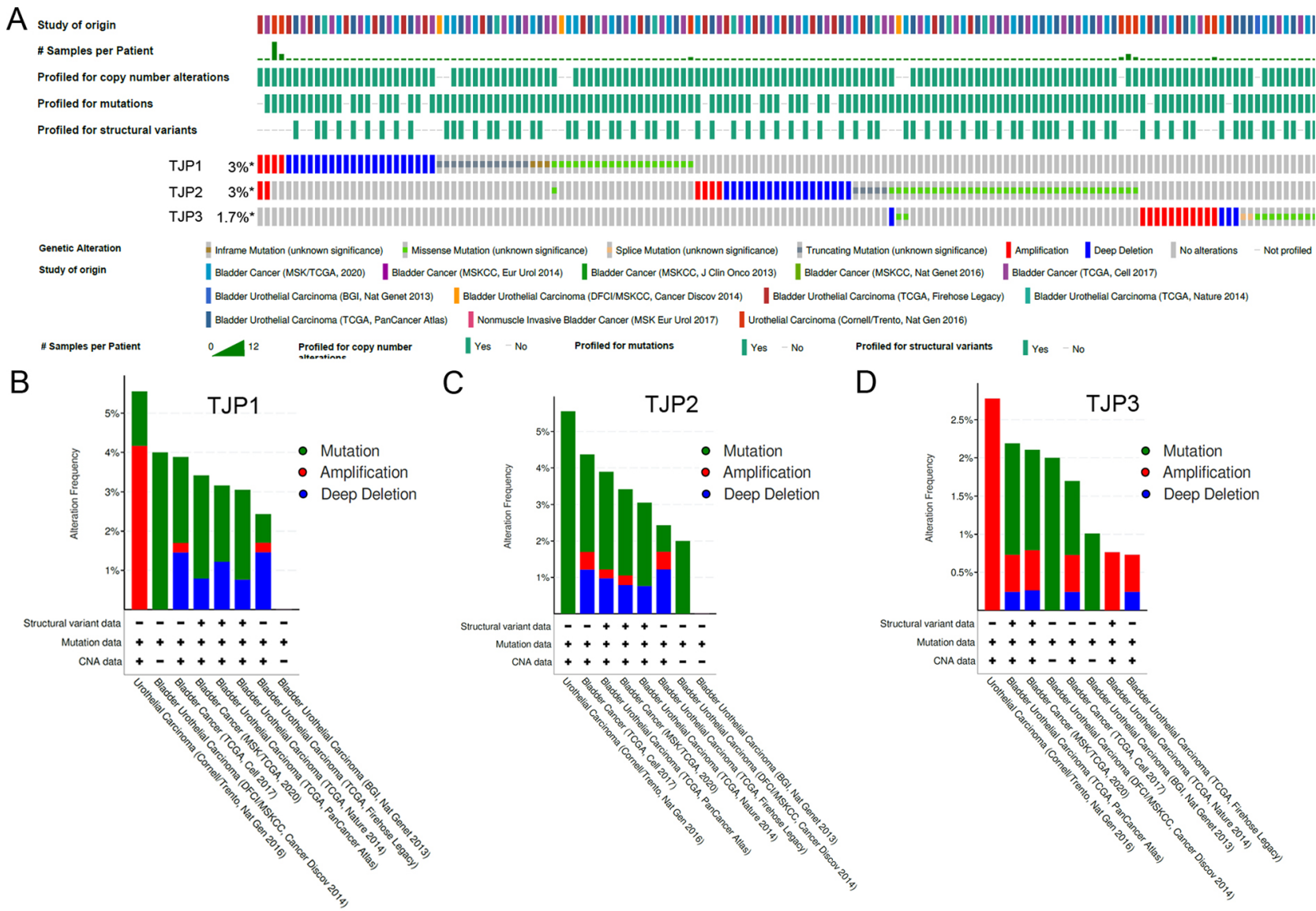

Figure 3. TJP1 amplification in bladder cancer. (A) Oncoprint showing TJP1, TJP2 and TJP3 genetic alterations in bladder cancer patients, respectively. Colors indicated type of genetic alteration (missense, inframe, truncated, amplification, deletion, fusion) and different cohort in the below the oncoprint. (B-D) Cancer type summary of TJP1 (B), TJP2 (C) and TJP3 (D) by different bladder cancer cohorts. CAN copy number alterations.

invasion abilities in the melanoma cell ${ }^{15}$. Specifically, high TJP1 mRNA expression has been reported in patients with bladder cancer. Knockdown of TJP1 inhibits cell proliferation, migration, and invasion in bladder cancer cell lines, while, TJP1 mRNA expression is associated with lymph node metastasis in bladder cancer patients ${ }^{16}$. Additionally, deletions and mutations of TJP1 promote cancer cell proliferation ${ }^{25}$. In our data showed expression of TJP1 protein was associated with grade and stage in patients with bladder cancer (Table 1).

Previous studies have shown that tumor mutational burden is a biomarker for predicting responsiveness to immune checkpoint blockade immunotherapy in several cancer types ${ }^{30,31}$. In our study, we found that TNT, TP53, and RYR3 mutations co-occurred with altered TJP1 in bladder cancer patients. In addition, TJP1 mRNA expression levels were positively correlated with TNT and RYR3 mRNA expression levels in patients with bladder cancer. We could not exclude the possibility that TJP1 amplification or expression is correlated with the response rate to immune checkpoint blockade; however, this is the first study to evaluate the TJP1 genetic alterations in bladder cancer patients. Accumulating evidence has shown genetic altered and expression correlated with chemotherapy response in bladder cancer ${ }^{19}$. We also found TJP2 and TJP3 mRNA expression positively correlated with chemoresistance in bladder cancer cell lines (Table 2). However, TJP1 mRNA expression was not significantly correlated with chemotherapy in bladder cancer cell lines. Further mechanisms are needed to investigate the underlying TJP1 expression and subsequently increased chemosensitivity in bladder cancer.

In conclusion, this study evaluated genetic variations in the TJP1 family by the amplification of TJP family members in bladder cancer patients. TJP1 protein expression correlated to tumor grade and stage, indicating that TJP1 can be used as an independent biomarker for bladder cancer staging.

\section{Materials and methods}

In silico genetic and mRNA profiles of the TJP1 family in multiple cancer cell lines and cancer patients. The mRNA expression levels and DNA copy numbers of TJP1, TJP2, and TJP3 in 40 different cancer cell lines were evaluated using the Cell Line Encyclopedia (CCLE) dataset. We stratified cancer cell types into upregulated (median $>0$ ) and downregulated (median $<0$ ). Genetic variations in the TJP1 family and genetic altered ranking in bladder cancer patients were evaluated using the online dataset (cBioPortal, v.3.6.20). 
A Negative control

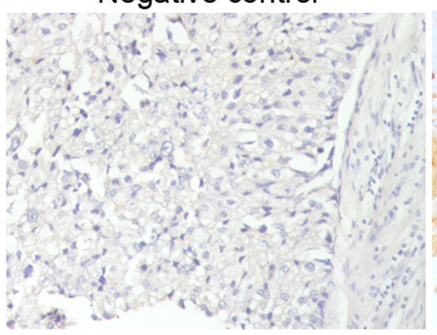

Tumor-Median expression

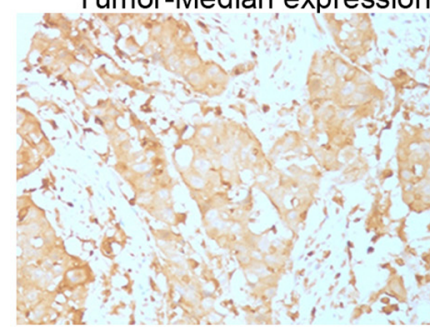

B

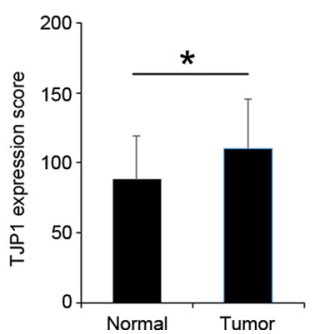

Normal

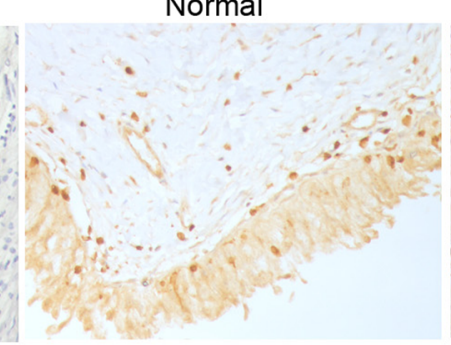

Tumor-High expression

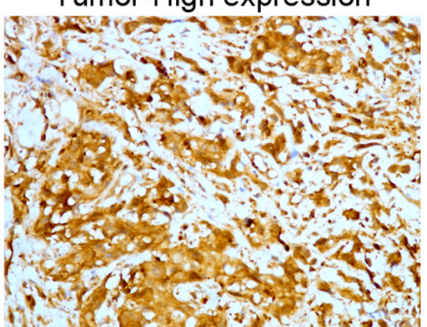

C

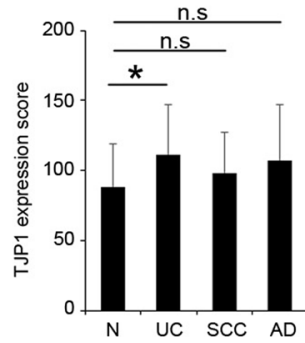

Figure 4. TJP1 upregulated in bladder cancer. (A) Representative IHC staining images of TJP1 in bladder cancer tissues. (B) Relative TJP1 protein levels in adjacent non-tumor tissue $(\mathrm{n}=16)$ and bladder cancer tissues $(n=192)$. (C) Relative TJP1 protein levels in urothelial carcinoma (UC, $n=160$ ), squamous cell (SCC, $n=16$ ) and adenocarcinoma $(\mathrm{AD}, \mathrm{n}=16)$ in bladder cancer tissues. Data are presented as the mean $\pm \mathrm{SD},{ }^{*} p<0.05 . n s$ not significant.

\begin{tabular}{|c|c|c|c|c|c|c|c|}
\hline \multirow[b]{3}{*}{ Variables } & \multirow[b]{3}{*}{ Item } & \multirow[b]{3}{*}{ Patient no. (\%) } & \multicolumn{4}{|c|}{ TJP-1 } & \multirow[b]{3}{*}{$p$ value } \\
\hline & & & \multicolumn{2}{|c|}{$\begin{array}{l}\text { Low } \\
(\leq 108.33)\end{array}$} & \multicolumn{2}{|c|}{\begin{tabular}{|l|} 
High \\
$(>108.33)$
\end{tabular}} & \\
\hline & & & No. & $\%$ & No. & $\%$ & \\
\hline & & $192(100.0)$ & 96 & 50.0 & 96 & 50.0 & \\
\hline \multirow{2}{*}{ Age (y) } & $\leq 60$ & $89(46.4)$ & 52 & 54.2 & 37 & 38.5 & 0.030 \\
\hline & $>60$ & $103(53.6)$ & 44 & 45.8 & 59 & 61.5 & \\
\hline \multirow{2}{*}{ Sex } & Female & $39(20.3)$ & 16 & 16.7 & 23 & 24.0 & 0.209 \\
\hline & Male & $153(79.7)$ & 80 & 83.3 & 73 & 76.0 & \\
\hline \multirow{3}{*}{ Grade } & I & $64(33.9)$ & 40 & 42.1 & 24 & 25.5 & 0.007 \\
\hline & II & $81(42.9)$ & 41 & 43.2 & 40 & 42.6 & \\
\hline & III & $44(23.3)$ & 14 & 14.7 & 30 & 31.9 & \\
\hline \multirow{2}{*}{ Stage } & $\mathrm{I} / \mathrm{II}$ & $154(80.2)$ & 84 & 87.5 & 70 & 72.9 & 0.011 \\
\hline & III/IV & $38(19.8)$ & 12 & 12.5 & 26 & 27.1 & \\
\hline \multirow{3}{*}{ Histology } & Urothelial carcinoma & $160(83.3)$ & 79 & 82.3 & 81 & 84.4 & 0.194 \\
\hline & Squamous cell carcinoma & $16(8.3)$ & 11 & 11.5 & 5 & 5.2 & \\
\hline & Adenocarcinoma & $16(8.3)$ & 6 & 6.2 & 10 & 10.4 & \\
\hline
\end{tabular}

Table 1. Correlation of TJP-1 expression with clinicopathological characteristics in bladder cancer. 

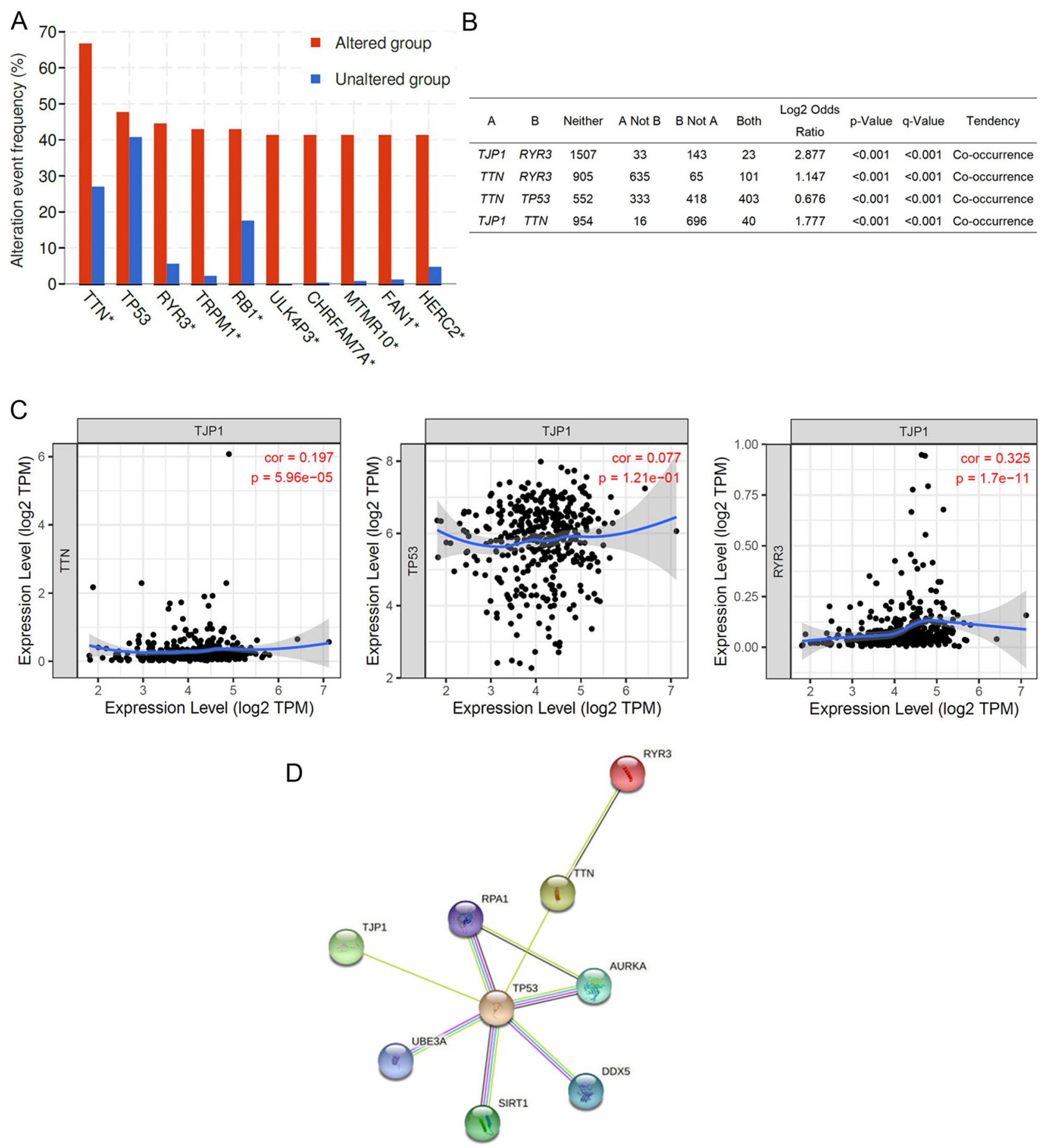

Figure 5. Correlation analysis of TJP1 and oncogenes in bladder cancer patients. (A) Frequency of genomic alteration in tumors from bladder cancer patients. ${ }^{\star} p<0.05$. (B) Major co-occurrences of genomic alterations of TJP1 and TTN, TP53 and RYR3 in bladder cancer patients. (C) Analysis of the correlation between TJP1 and TTN/TP53/RYR3 mRNA expression using TIMER. (D) STRING protein-protein interaction networks for TJP1, TP53, TTN and RYR3.

Patients and specimen collection. Bladder tumor tissue was collected from 192 patients with bladder cancer (sample from 160 patients with urothelial carcinoma, 16 patients with squamous cell and 16 patients with adenocarcinoma). The bladder cancer tissue array (\#BL2081a) was purchased from Biomax (Rockville, MD, USA) and was used for immunohistochemical (IHC) staining to evaluate the expression of TJP1 protein. The pathologic grade was classified based on the World Health Organization (WHO) histological criteria ${ }^{32}$. 


\begin{tabular}{|l|l|l|l|l|l|l|}
\hline \multirow{2}{*}{ Drugs } & \multicolumn{4}{|l|}{ TJP1 } & TJP2 & \multicolumn{2}{l|}{ TJP3 } \\
\cline { 2 - 7 } & Correlation & $\boldsymbol{p}$-value & Correlation & $\boldsymbol{p}$-value & Correlation & $\boldsymbol{p}$-value \\
\hline Cisplatin & 0.427 & 0.095 & 0.591 & $0.028^{*}$ & 0.345 & 0.149 \\
\hline Doxorubicin & 0.082 & 0.405 & 0.264 & 0.217 & 0.145 & 0.335 \\
\hline Epirubicin & -0.082 & 0.405 & -0.127 & 0.355 & 0.055 & 0.437 \\
\hline Gemcitabine & -0.200 & 0.278 & 0.291 & 0.193 & 0.336 & 0.156 \\
\hline Mitomycin C & 0.373 & 0.129 & 0.291 & 0.193 & 0.555 & $0.038^{*}$ \\
\hline
\end{tabular}

Table 2. Correlation of TJP family expression with chemotherapy drugs in bladder cancer cell lines. ${ }^{\star} p$ values $<0.05$ were considered statistically significant.

Immunohistochemical staining. IHC staining was performed according to the manufacturer's instructions. The primary antibody of TJP1 (\#HPA001636, 1:50) was purchased from Sigma-Aldrich (St. Louis, MO, USA). TJP1 expression was evaluated using the $\mathrm{H}$-score, which is calculated as the percentage of positively stained cells multiplied by the staining intensity. In this study, two physicians (Y.-C L and J.-B L) were used to objectively evaluate the scores.

Correlation of TJP1 with other high frequency mutation genes. The genetic alteration, co-occurrence, and mRNA expression levels of high-frequency mutation genes (TTN, RYR3, TRPM1, RB1, ULK4P3, CHRFAM7A, FAN1, and HERC2) correlating to TJP1 expression were evaluated using TIMER.

Correlation of TJP family with chemotherapy drugs. TJP family mRNA levels downloaded from depmap portal (https://depmap.org/portal/download/?release=CCLE+2019\&release=Fusion\&release=DNA+ Copy+Number). The IC50 of cisplatin, doxorubicin, epirubicin, gemcitabine, mitomycin C was downloaded from Genomics of Drug Sensitivity in Cancer database (https://www.cancerrxgene.org/). The bladder cancer cell lines include 5637, 639V, 647V, HT1197, HT1376, KU1919, RT-122, T24, TDDSUP, UMUC3, and VMCUB1.

Statistical analyses. All statistical analyses were performed using SPSS (version 19.0; IBM, Armonk, NY, USA). The Chi-squared test was used to determine the correlation between TJP1 expression and the tumor stage, grade, size, and the patient's age at diagnosis. The Student's $t$ test was used to identify significant differences between the treatment groups. Statistical significance was set at $p<0.05$.

Received: 28 September 2021; Accepted: 10 January 2022

Published online: 27 January 2022

\section{References}

1. Soukup, V. et al. Prognostic performance and reproducibility of the 1973 and 2004/2016 World Health Organization grading classification systems in non-muscle-invasive bladder cancer: A European Association of Urology Non-muscle Invasive Bladder Cancer Guidelines Panel Systematic Review. Eur. Urol. 72, 801-813. https://doi.org/10.1016/j.eururo.2017.04.015 (2017).

2. van Rhijn, B. W. et al. A new and highly prognostic system to discern T1 bladder cancer substage. Eur. Urol. 61, 378-384. https:// doi.org/10.1016/j.eururo.2011.10.026 (2012).

3. Metts, M. C., Metts, J. C., Milito, S. J. \& Thomas, C. R. Jr. Bladder cancer: A review of diagnosis and management. J. Natl. Med. Assoc. 92, 285-294 (2000).

4. Babjuk, M. Trends in bladder cancer incidence and mortality: Success or disappointment?. Eur. Urol. 71, 109-110. https://doi.org/ 10.1016/j.eururo.2016.06.040 (2017).

5. Sun, M. \& Trinh, Q. D. Diagnosis and staging of bladder cancer. Hematol. Oncol. Clin. North Am. 29, 205-218, vii. https://doi.org/ 10.1016/j.hoc.2014.10.013 (2015).

6. Bray, F. et al. Global cancer statistics 2018: GLOBOCAN estimates of incidence and mortality worldwide for 36 cancers in 185 countries. CA Cancer J. Clin. 68, 394-424. https://doi.org/10.3322/caac.21492 (2018).

7. Patel, V. G., Oh, W. K. \& Galsky, M. D. Treatment of muscle-invasive and advanced bladder cancer in 2020. CA Cancer J. Clin. 70, 404-423. https://doi.org/10.3322/caac.21631 (2020).

8. Cheng, C. Y. \& Mruk, D. D. The blood-testis barrier and its implications for male contraception. Pharmacol. Rev. 64, 16-64. https:// doi.org/10.1124/pr.110.002790 (2012).

9. Gibson, M. C. \& Perrimon, N. Apicobasal polarization: Epithelial form and function. Curr. Opin. Cell Biol. 15, 747-752. https:// doi.org/10.1016/j.ceb.2003.10.008 (2003).

10. Marchiando, A. M., Graham, W. V. \& Turner, J. R. Epithelial barriers in homeostasis and disease. Annu. Rev. Pathol. 5, 119-144. https://doi.org/10.1146/annurev.pathol.4.110807.092135 (2010).

11. Abraham, V. et al. Heterocellular gap junctional communication between alveolar epithelial cells. Am. J. Physiol. Lung Cell. Mol. Physiol. 280, L1085-L1093. https://doi.org/10.1152/ajplung.2001.280.6.L1085 (2001).

12. Guillemot, L., Paschoud, S., Pulimeno, P., Foglia, A. \& Citi, S. The cytoplasmic plaque of tight junctions: A scaffolding and signalling center. Biochim. Biophys. Acta 1778, 601-613. https://doi.org/10.1016/j.bbamem.2007.09.032 (2008).

13. Itoh, M. et al. Direct binding of three tight junction-associated MAGUKs, ZO-1, ZO-2, and ZO-3, with the COOH termini of claudins. J. Cell Biol. 147, 1351-1363. https://doi.org/10.1083/jcb.147.6.1351 (1999).

14. Doi, Y. et al. VEGF-A/VEGFR-2 signaling plays an important role for the motility of pancreas cancer cells. Ann. Surg. Oncol. 19, 2733-2743. https://doi.org/10.1245/s10434-011-2181-6 (2012). 
15. Smalley, K. S. et al. Up-regulated expression of zonula occludens protein-1 in human melanoma associates with N-cadherin and contributes to invasion and adhesion. Am. J. Pathol. 166, 1541-1554. https://doi.org/10.1016/S0002-9440(10)62370-X (2005).

16. Tsai, K. W., Kuo, W. T. \& Jeng, S. Y. Tight junction protein 1 dysfunction contributes to cell motility in bladder cancer. Anticancer Res. 38, 4607-4615. https://doi.org/10.21873/anticanres.12765 (2018).

17. Li, T. et al. TIMER: A web server for comprehensive analysis of tumor-infiltrating immune cells. Cancer Res. 77, e108-e110. https:// doi.org/10.1158/0008-5472.CAN-17-0307 (2017).

18. Szklarczyk, D. et al. STRING v11: Protein-protein association networks with increased coverage, supporting functional discovery in genome-wide experimental datasets. Nucleic Acids Res. 47, D607-D613. https://doi.org/10.1093/nar/gky1131 (2019).

19. Mari, A. et al. Genetic determinants for chemo- and radiotherapy resistance in bladder cancer. Transl. Androl. Urol. 6, 1081-1089. https://doi.org/10.21037/tau.2017.08.19 (2017).

20. Itoh, M., Nagafuchi, A., Moroi, S. \& Tsukita, S. Involvement of ZO-1 in cadherin-based cell adhesion through its direct binding to alpha catenin and actin filaments. J. Cell Biol. 138, 181-192. https://doi.org/10.1083/jcb.138.1.181 (1997).

21. Kleeff, J. et al. Altered expression and localization of the tight junction protein ZO-1 in primary and metastatic pancreatic cancer. Pancreas 23, 259-265. https://doi.org/10.1097/00006676-200110000-00006 (2001).

22. Ni, S. et al. Increased ZO-1 expression predicts valuable prognosis in non-small cell lung cancer. Int. J. Clin. Exp. Pathol. 6, 2887-2895 (2013).

23. Balda, M. S. \& Matter, K. Tight junctions and the regulation of gene expression. Biochim. Biophys. Acta 1788, 761-767. https:// doi.org/10.1016/j.bbamem.2008.11.024 (2009).

24. Polette, M. et al. Beta-catenin and ZO-1: Shuttle molecules involved in tumor invasion-associated epithelial-mesenchymal transition processes. Cells Tissues Organs 185, 61-65. https://doi.org/10.1159/000101304 (2007).

25. Willott, E. et al. The tight junction protein ZO-1 is homologous to the Drosophila discs-large tumor suppressor protein of septate junctions. Proc. Natl. Acad. Sci. U.S.A. 90, 7834-7838. https://doi.org/10.1073/pnas.90.16.7834 (1993).

26. Hoover, K. B., Liao, S. Y. \& Bryant, P. J. Loss of the tight junction MAGUK ZO-1 in breast cancer: relationship to glandular differentiation and loss of heterozygosity. Am. J. Pathol. 153, 1767-1773. https://doi.org/10.1016/S0002-9440(10)65691-X (1998).

27. Kaihara, T. et al. Redifferentiation and ZO-1 reexpression in liver-metastasized colorectal cancer: possible association with epidermal growth factor receptor-induced tyrosine phosphorylation of ZO-1. Cancer Sci. 94, 166-172. https://doi.org/10.1111/j. 1349-7006.2003.tb01414.x (2003).

28. Li, G., Satyamoorthy, K. \& Herlyn, M. N-cadherin-mediated intercellular interactions promote survival and migration of melanoma cells. Cancer Res. 61, 3819-3825 (2001)

29. Tran, N. L., Adams, D. G., Vaillancourt, R. R. \& Heimark, R. L. Signal transduction from N-cadherin increases Bcl-2. Regulation of the phosphatidylinositol 3-kinase/Akt pathway by homophilic adhesion and actin cytoskeletal organization. J. Biol. Chem. 277, 32905-32914. https://doi.org/10.1074/jbc.M200300200 (2002).

30. Yarchoan, M., Hopkins, A. \& Jaffee, E. M. Tumor mutational burden and response rate to PD-1 inhibition. N. Engl. J. Med. 377, 2500-2501. https://doi.org/10.1056/NEJMc1713444 (2017).

31. Samstein, R. M. et al. Tumor mutational load predicts survival after immunotherapy across multiple cancer types. Nat. Genet. 51, 202-206. https://doi.org/10.1038/s41588-018-0312-8 (2019).

32. Humphrey, P. A., Moch, H., Cubilla, A. L., Ulbright, T. M. \& Reuter, V. E. The 2016 WHO classification of tumours of the urinary system and male genital organs-part B: Prostate and bladder tumours. Eur. Urol. 70, 106-119. https://doi.org/10.1016/j.eururo. 2016.02.028 (2016).

\section{Acknowledgements}

This study was supported by grants from the Kaohsiung Veterans General Hospital, Taiwan [VGHKS109-055 (W.T.K), KSVGH110-043 (W.T.K) and KSVGH110-144 (Y.F.Y), Kaohsiung Medical University Research Foundation [KMU-M108004 (Y.C.L) and KMU-M109015 (Y.C.L)], Yen Tjing Ling Medical Foundation [CI-111-7 (Y.F.Y) and CI-111-9 (Y.C.C)], Veterans General Hospitals and University System of Taiwan Joint Research Program [VGHUST111-G3-3-1 (Y.F.Y) and VGHUST111-G3-3-2 (Y.C.C)], and National Science Council [MOST 110-2314-B-075B-009-MY3 (Y.F.Y)].

\section{Author contributions}

Wrote manuscript, Y.-F.Y., conducted experiments, provide materials and reagents, K.-W.T., W.-T.K., performed the data analysis, Y.-C.L. and Y.-C.C, performed IHC and IHC scoring, Y.-C.L. and J.-B.L., designed the project, Y.-F.Y., K.-W.T. All authors have read and agreed to the published version of the manuscript.

\section{Competing interests}

The authors declare no competing interests.

\section{Additional information}

Supplementary Information The online version contains supplementary material available at https://doi.org/ 10.1038/s41598-022-05631-y.

Correspondence and requests for materials should be addressed to Y.-F.Y.

Reprints and permissions information is available at www.nature.com/reprints.

Publisher's note Springer Nature remains neutral with regard to jurisdictional claims in published maps and institutional affiliations. 
(c) (i) Open Access This article is licensed under a Creative Commons Attribution 4.0 International cc) License, which permits use, sharing, adaptation, distribution and reproduction in any medium or format, as long as you give appropriate credit to the original author(s) and the source, provide a link to the Creative Commons licence, and indicate if changes were made. The images or other third party material in this article are included in the article's Creative Commons licence, unless indicated otherwise in a credit line to the material. If material is not included in the article's Creative Commons licence and your intended use is not permitted by statutory regulation or exceeds the permitted use, you will need to obtain permission directly from the copyright holder. To view a copy of this licence, visit http://creativecommons.org/licenses/by/4.0/.

(C) The Author(s) 2022 